\title{
O-Aryl-Glycoside Ice Recrystallization Inhibitors as Novel Cryoprotectants: A Structure-Function Study
}

Chantelle J. Capicciotti, $^{\dagger}$ Ross S. Mancini, ${ }^{\dagger}$ Tracey R. Turner, ${ }^{\dagger}$ Toshie Koyama, $^{\S}$ Matthew G. Alteen, ${ }^{\dagger}$ Malay Doshi, ${ }^{\dagger}$ Takaaki Inada, ${ }^{\S}$ Jason P. Acker, ${ }^{\dagger}$ and Robert N. Ben*, ${ }^{\dagger}$

${ }^{\dagger}$ Department of Chemistry, University of Ottawa, D’Iorio Hall, 10 Marie Curie, Ottawa, Ontario K1N 6N5, Canada

${ }^{\ddagger}$ Canadian Blood Services, Centre for Innovation, 8249-114 Street NW, Edmonton, Alberta T6G 2R8, Canada

${ }^{\S}$ National Institute of Advanced Industrial Science and Technology (AIST), 1-2-1 Namiki, Tsukuba, Ibaraki 305-8564, Japan

Supporting Information

ABSTRACT: Low-molecular-weight ice recrystallization inhibitors (IRIs) are ideal cryoprotectants that control the growth of ice and mitigate cell damage during freezing. Herein, we describe a detailed study correlating the ice recrystallization inhibition activity and the cryopreservation ability with the structure of $\mathrm{O}$-aryl-glycosides. Many effective IRIs are efficient cryoadditives for the freezing of red blood cells (RBCs). One effective cryoadditive did not inhibit ice recrystallization but instead inhibited ice nucleation, demonstrating the significance of inhibiting both processes and illustrating the importance of this emerging class of cryoprotectants.

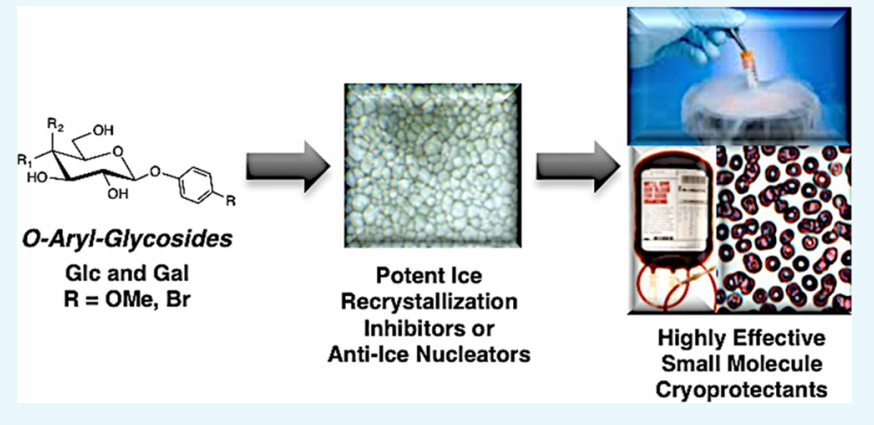

amount of glycerol (and DMSO) will greatly improve the logistical issues associated with managing inventories of frozen cells and tissues, making these more accessible for lifesaving therapies.

Ice recrystallization is a significant contributor to cellular damage and death during the freezing and thawing steps of the cryopreservation process. ${ }^{13-15}$ Thus, there is tremendous interest in designing compounds that are effective ice recrystallization inhibitors (IRIs) to mitigate this damage and improve cellular viability and function post-thaw. ${ }^{16-20}$ Antifreeze (glyco)proteins $[\mathrm{AF}(\mathrm{G}) \mathrm{Ps}]$ are nature's most potent IRIs; ${ }^{21-23}$ however, they often fail as cryoprotectants because of their ability to bind to ice and alter the ice crystal habit, which is detrimental to cells. ${ }^{24-27}$ Large peptides and glycopeptide analogues of $\mathrm{AF}(\mathrm{G}) \mathrm{Ps}^{28-30}$ and high-molecular-weight polymers ${ }^{18,31-33}$ such as poly(vinyl alcohol) (PVA) have also been explored as cryoprotectants. However, small molecules with molecular masses less than 300 atomic mass units are highly attractive alternatives that can be efficiently synthesized and are amenable to large-scale preparations. Furthermore, there is less likelihood of nonspecific toxic effects and immunogenic responses that can be associated with peptides or proteins.

Prior work by our group has demonstrated that small amphiphilic carbohydrate-based and amino acid-based molecules can be very effective IRIs. ${ }^{34-36}$ Recently, our group has reported two novel glucose-based $O$-aryl-glycosides, $\beta$-PMP-

Received: August 1, 2016

Accepted: September 14, 2016

Published: October 24, 2016 

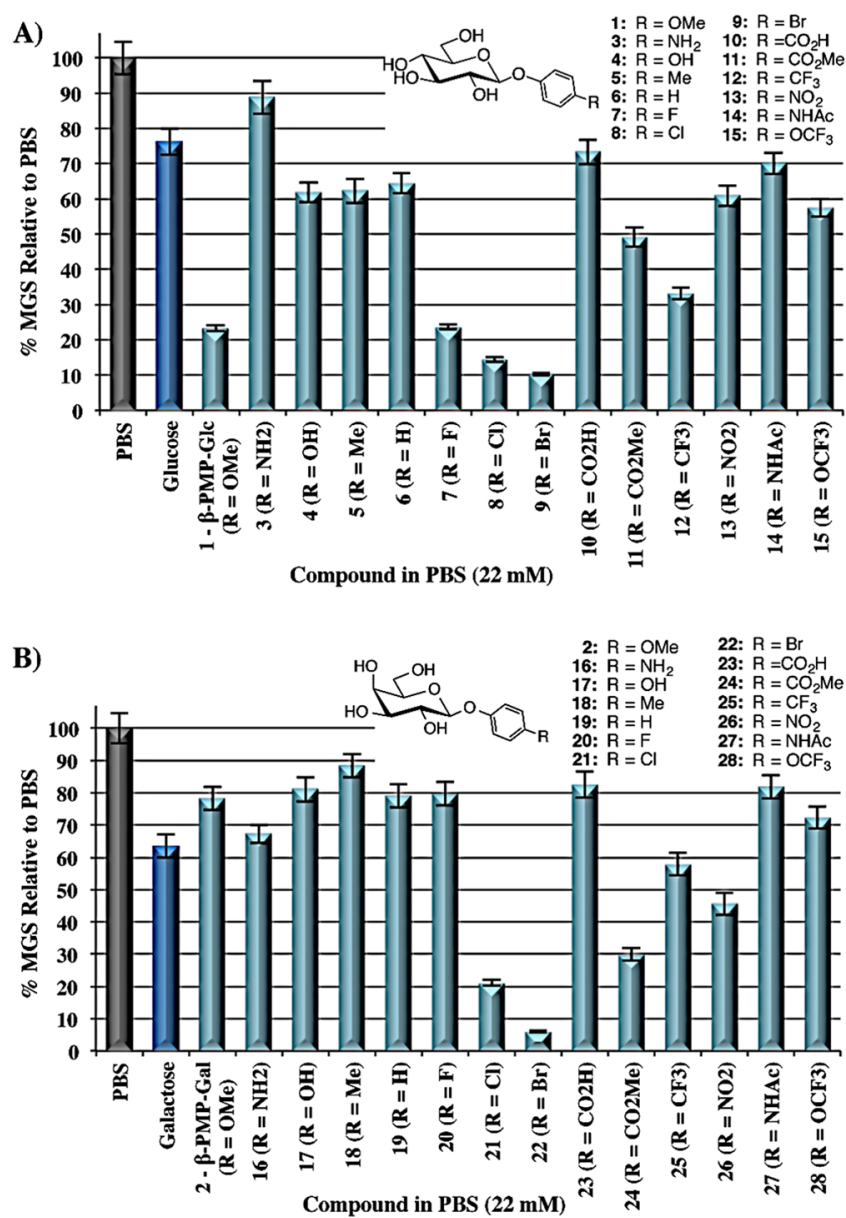

Figure 1. Structures and IRI activity of $\beta$-linked para-substituted (A) $\beta$-PMP-Glc (1) and $O$-aryl-glucosides 3-15 and (B) $\beta$-PMP-Gal (2) and $O$-aryl-galactosides 16-28. All compounds were assessed at 22 $\mathrm{mM}$ in PBS. IRI activity is represented as \% MGS relative to the PBS positive control. The error bars indicate standard error of the mean (SEM).

Glc (1) and $\beta$-pBrPh-Glc (9) (Figure 1A), bearing a parasubstituted aryl moiety as the hydrophobic component, which are very effective IRIs. ${ }^{37}$ Furthermore, these compounds are effective cryoprotectants for human RBCs, furnishing high postthaw yields using only $15 \%$ glycerol and not $40 \%$, resulting in shorter post-thaw deglycerolization times. This is the first example where a small molecule IRI was used as a cryoprotectant. Herein, we report a detailed investigation into the structural features important for the IRI activity and cryopreservation ability of this novel class of $\mathrm{O}$-aryl-glycoside ice recrystallization inhibitor.

\section{RESULTS AND DISCUSSION}

$\beta$-PMP-Glc (1) is a glucose-based derivative bearing a paramethoxyphenyl (PMP) moiety and is an effective inhibitor of ice recrystallization. However, the galactose analogue $\beta$-PMPGal (2) exhibits very weak activity (Figure 1). ${ }^{37}$ This was surprising because previous reports from our laboratory described that pyranose-based derivatives containing a more hydrated galactose head group were better IRIs than those with less hydrated glucose head groups. ${ }^{29,34,38}$ To further explore this, we studied the influence of the para-substituent on activity by substituting this position with various atoms and functional groups. The IRI activity of the glucose-based (3-15) and the galactose-based (16-28) derivatives was determined using a "splat-cooling assay" (Figure 1). ${ }^{39,40}$ IRI activity is represented as a ratio of the mean grain size (MGS) of ice crystals of a given sample to that of a phosphate-buffered saline (PBS) solution, with smaller percent MGS (\% MGS) values indicative of smaller ice crystals and more effective inhibition.

Many of the $\mathrm{O}$-aryl-glucosides exhibit moderate to weak activity (Figure 1A), and most of them are less active (larger crystals) than $\beta$-PMP-Glc (1). Substitution of the para-methoxy moiety with halogens $(-\mathrm{F},-\mathrm{Cl}$, or $-\mathrm{Br}, 7-9)$ or a trifluoromethyl group $\left(-\mathrm{CF}_{3}, 12\right)$ resulted in similar or better IRI activity, with the most active compound being $\beta$-pBrPh-Glc (9). The para-iodo derivative could be assessed only at its maximum solubility of $5.5 \mathrm{mM}$, and it was inactive (data not shown). It is evident that the methyl group of the $-\mathrm{OMe}$ is crucial for the activity of $\beta$-PMP-Glc because replacement of this moiety with an $-\mathrm{OH}$ or $-\mathrm{OCF}_{3}$ (4 and 15) group decreased activity. An oxygen atom connecting the aryl ring and the methyl group is also important because 5 resulted in a similar reduction in activity.

The $O$-aryl-galactosides exhibit a very different activity profile than the glucosides. Most galactosides had weak activity that was much less than that of galactose (Figure 1B). Potent activity was observed with $-\mathrm{Cl}$ and $-\mathrm{Br}$ substituents (21-22); however, the fluoro-derivative $\mathbf{2 0}$ was inactive. In general, the galactosides were less active than the corresponding glucose derivatives. Exceptions include 16, 24, and 26 with a para$\mathrm{NH}_{2},-\mathrm{CO}_{2} \mathrm{Me}$, or $-\mathrm{NO}_{2}$ group, compared with analogous glucose derivatives $3, \mathbf{1 1}$, and 13. Overall, it is evident that electronic effects do not influence the ice recrystallization inhibition ability of the $\mathrm{O}$-aryl-glycosides in any obvious manner. In the glucoside series, $-\mathrm{OMe},-\mathrm{OH}$, and $-\mathrm{NH}_{2}$ groups $(1,3-4)$ are all electron-donating groups (EDGs), yet 1 is highly active, whereas 3 is the least active glucose derivative. Moreover, both $-\mathrm{NO}_{2}$ and $-\mathrm{CF}_{3}$ groups are strong electronwithdrawing groups (EWGs); however, the $-\mathrm{CF}_{3}$ derivative 12 was highly active, whereas the $-\mathrm{NO}_{2}$ derivative 13 was only moderately active. In the galactoside series, none of the derivatives with EDGs were active (2, 16-19). The activity of derivatives with EWGs varied from weak (20 and 23) to moderate (25-26) with only 24 exhibiting high activity.

Thus far, in this class of compounds, one of the best IRIs is $\beta$-PMP-Glc (1), and it appears that substitution at the paraposition with anything other than a methoxy or a halogen group is detrimental for activity. We next sought to explore other derivatives of the $\beta$-PMP-Glc structure to determine other structural features that are important for this potent activity (Figure 2). This included exploring the $\mathrm{C} 1$ glycosidic bond stereochemistry (29-34), the effect of methoxy group regiochemistry (35-36), and the importance of an $O$-linkage (37-39) at C1. Unfortunately, all of these modifications were detrimental to IRI activity. An $\alpha$-linkage greatly reduced the activity (30), a trend that was observed also with the $\alpha$-linked halogen derivatives 33-34, highlighting the importance of a $\beta$ linkage. Similarly, a methoxy group in the para-position was essential as meta- and ortho-derivatives 35-36 displayed moderate activity. It is evident that modifying the $O$-glycosidic linkage is also detrimental because $\mathrm{N}$ - and S-linked PMP derivatives 37-38 had drastically decreased the activity. In addition, a direct connection of the aryl ring to the $\mathrm{C} 1$ oxygen is crucial because the para-methoxybenzyl derivative 39 was also inactive. Finally, conjugating the O-PMP group to the C6 position (40) rather than $C 1$ also resulted in weak activity. 


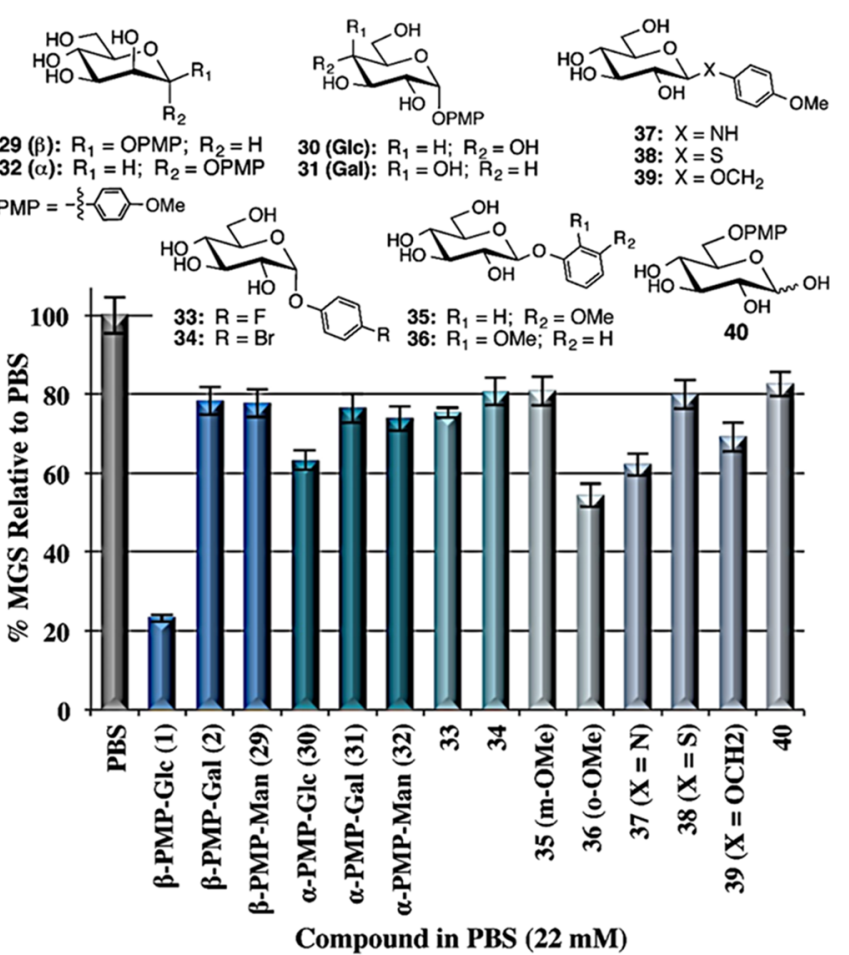

Figure 2. Structures and IRI activity (\% MGS relative to PBS) of $\alpha$ and $\beta$-linked PMP-glycoside derivatives 29-40. The error bars indicate SEM.

The IRI activity of $\beta$-PMP-Glc is highly sensitive to the relative orientation of the hydroxyl groups on the pyranose ring. Equatorial $\mathrm{C} 2$ and $\mathrm{C} 4$ hydroxyl groups are required because $\beta$-PMP-Gal (2) and $\beta$-PMP-Man (29) were weakly active (Figure 2). Furthermore, the C6-hydroxymethyl group is also important because removing this group (xylose derivative 41), methylating this position (42), and extending this position by one carbon (43) diminished activity (Figure 3). Glycosylating the $\mathrm{C} 6$ position of $\beta$-PMP-Glc resulted in a similar decrease in activity, and this was independent of the glycosidic-bond stereochemistry or whether the conjugated monosaccharide was galactose or glucose (44-46). Interestingly, glycosylation at the $\mathrm{C} 4$ position appears to be tolerated with little change in activity. $\beta$-PMP-lactose 47 had better activity than disaccharides 44-46, although its activity was diminished compared with that of the parent $\beta$-PMP-Glc. Collectively, these results highlight the importance of the various hydroxyl groups on the pyranose ring of $\beta$-PMP-Glc and how the $\mathrm{C6}-\mathrm{OH}$ is particularly sensitive to modifications.

Other disaccharides assessed for activity were the dihydroxybenzene-linked disaccharides 48-50. These were 1,1-linked using an $O$-phenyl group in which the para-substituent was a second $\beta$-linked galactose or glucose residue (Figure 3). Interestingly, disaccharide $\mathbf{4 8}$ containing two $\beta$-linked glucose units had potent IRI activity that was slightly less than that of $\beta$ PMP-Glc. Thus, it appears that replacing the para-methoxy substituent with another glucose residue retains activity. Conversely, when the para-substituent was a galactose unit (49), only moderate IRI activity was observed. Disaccharide 50 containing two galactose units had weak activity that was similar to that of the monosaccharide $\beta$-PMP-Gal (2), indicating that the additional galactose unit had no effect on ice recrystallization inhibition.
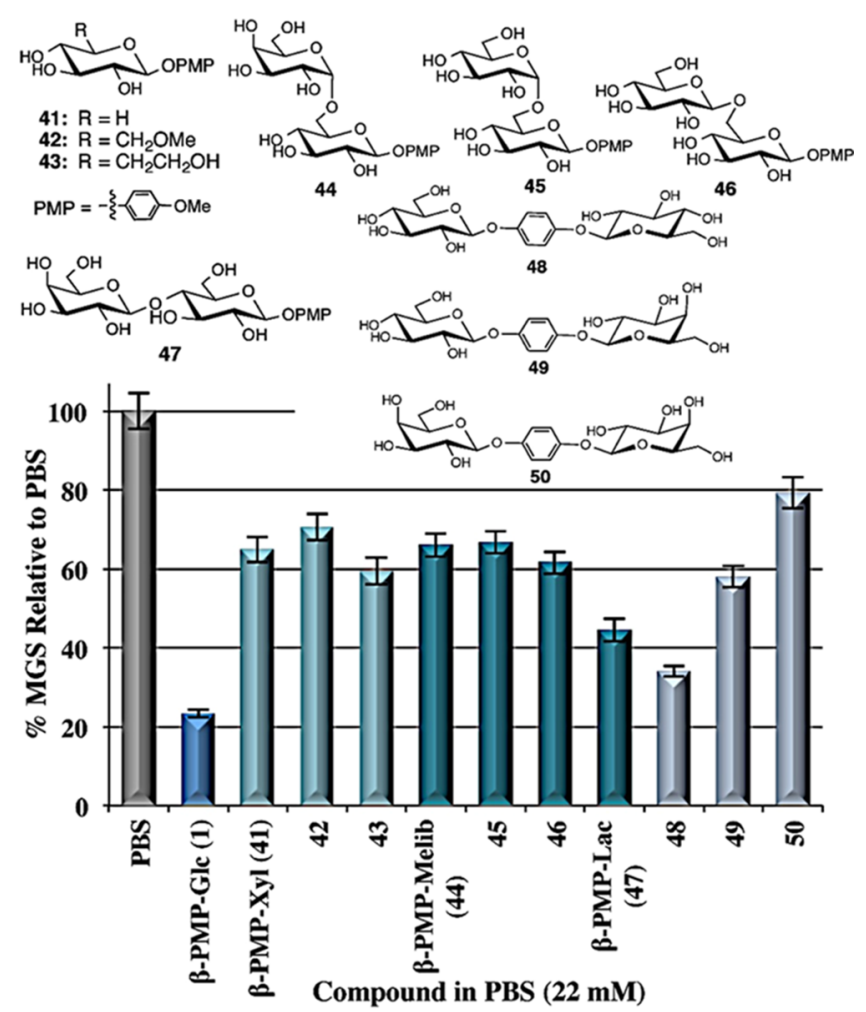

Figure 3. Structures and IRI activity (\% MGS relative to PBS) of $\beta$ PMP-Glc derivatives 41-43 and $\beta$-PMP-disaccharide derivatives 4450. The error bars indicate SEM.

Overall, the ice recrystallization inhibition data of the $O$-arylglycosides uncovered a few compounds in this class of molecules that are highly efficient IRIs. Having previously reported that both $\beta$-PMP-Glc (1) and $\beta$-pBrPh-Glc (9) are also effective cryoadditives for $\mathrm{RBCs},{ }^{37}$ we investigated the cryoprotective ability of many of these derivatives. This would provide insight into the structural features of $\beta$-PMP-Glc that are beneficial for its cryoprotective ability and would also allow us to explore the relationship between ice recrystallization inhibition and the cryopreservation ability for this class of compounds. Two freezing protocols were used to cryopreserve human RBCs in cryoprotective solutions containing a reduced quantity of glycerol (15\%) with or without the $O$-aryl-glycoside additives. A two-step slow-freezing protocol was used where samples were cooled at a rate of $1{ }^{\circ} \mathrm{C} / \mathrm{min}$ to $-40{ }^{\circ} \mathrm{C}$, then immediately stored at $-80{ }^{\circ} \mathrm{C}$ before thawing., ${ }^{8,41}$ This is representative of the current clinical $\mathrm{RBC}$ cryopreservation protocols used in North America with high glycerol concentrations $(40 \%))^{3,12,42,43}$ A rapid-freezing protocol was also used where samples were dumped into dry ice $\left(-80{ }^{\circ} \mathrm{C}\right)$ to achieve rapid freezing rates. ${ }^{3,4,44} \mathrm{O}$-Aryl-glycoside derivatives were assessed at concentrations previously determined to be most effective for $\beta$-PMP-Glc (1) and $\beta$-pBrPh-Glc (9). ${ }^{37}$ Postthaw hemolysis was quantified using the Drabkin's assay. ${ }^{45,46}$ RBC freezing results (Figure 4) are reported as a percentage of post-thaw integrity (100 - \% hemolysis). O-Aryl-glycosides that have a potent IRI activity are indicated by a diamond ( $)$ to easily identify the RBC freezing results of these compounds.

With the slow-freezing protocol, $110 \mathrm{mM} \beta$-PMP-Glc in $15 \%$ glycerol doubles the post-thaw RBC integrity (50\%) of the $15 \%$ glycerol control (25\%). Other derivatives with potent IRI activity, such as $\beta$-pFPh-Glc (7) and $\beta$-pBrPh-Glc (9), 

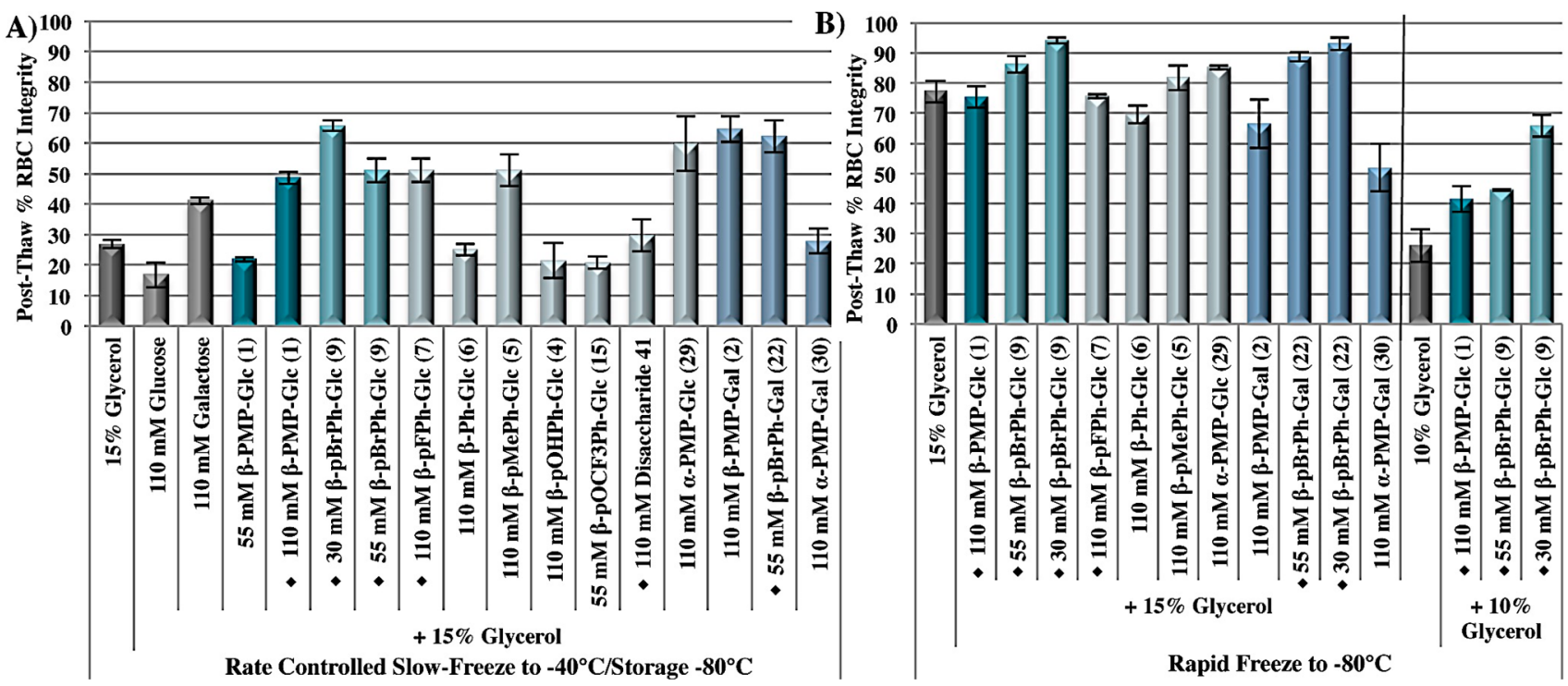

Figure 4. Post-thaw RBC integrity after freezing using $\mathrm{O}$-aryl-glycoside IRIs in $15 \%$ glycerol cryosolutions. (A) Rate-controlled slow-freezing at 1 ${ }^{\circ} \mathrm{C} / \mathrm{min}$ to $-40{ }^{\circ} \mathrm{C}$ followed by $-80{ }^{\circ} \mathrm{C}$ storage. (B) Rapid-freezing to $-80{ }^{\circ} \mathrm{C}$ by plunging into solid $\mathrm{CO}_{2}$ (dry ice). All samples were thawed at 37 ${ }^{\circ} \mathrm{C}$, and post-thaw RBC hemolysis was determined using the Drabkin's assay. Results are presented as mean post-thaw \% RBC integrity (100 - \% hemolysis). The error bars represent SEM. indicates compounds with a potent IRI activity.

furnished similar results. It is interesting to note that 9 is more effective at $30 \mathrm{mM}$ than at $55 \mathrm{mM}$, suggesting that toxicity may be an issue with this particular compound. Disaccharide 48, which was a moderate inhibitor of ice recrystallization, failed to increase post-thaw integrity and was worse than $15 \%$ glycerol. Many of the less-active glucosides failed to give post-thaw integrity higher than the glycerol control $(25 \%-30 \%, 4,6,15)$. Surprisingly, $\beta$-pMePh-Glc (5) and $\alpha$-PMP-Glc (30) had similar post-thaw integrities as $\beta$-PMP-Glc $(50 \%-60 \%)$ despite being less active. It appears that simply the presence of the methyl group either directly connected to the aryl ring (5) or linked through an oxygen atom (1) is sufficient for RBC freezing, and removal of this group (with 4 and 6) abolishes any cryoprotective effect. In contrast to ice recrystallization inhibition, cryoprotective ability is not sensitive to $\mathrm{C} 1$ glycosidic-bond stereochemistry because $\alpha$ - and $\beta$-PMP-Glc had similar post-thaw RBC integrity. When $O$-aryl-galactosides were used as cryoadditives, $\beta$-PMP-Gal (2) in $15 \%$ glycerol resulted in a slightly better post-thaw integrity than $\beta$-PMP-Glc $(65 \%)$, despite the fact that this compound was not IRI active. In contrast to the PMP-Glc derivatives, the cryoprotective ability of the Gal derivatives is sensitive to the stereochemistry of the glycosidic-bond because $\alpha$-PMP-Gal (31) resulted in a low post-thaw integrity similar to $15 \%$ glycerol. The fact that 2 and 9 were effective cryoprotectants, yet $\mathbf{2}$ was not IRI active, was a very surprising and unexpected result. Subsequent experiments have verified that although 2 does not exhibit ice recrystallization inhibition it is a very effective inhibitor of ice nucleation. ${ }^{47} \beta$-PMP-Gal (2) results in a significantly higher percentage of unfrozen droplets of water when supercooled to $-37{ }^{\circ} \mathrm{C}$ (Figure 5), inactivating the ice-nucleating agent AgI. This result could explain the ability of $\mathbf{2}$ to increase the number of post-thaw RBCs because when nucleation is inhibited, a greater degree of supercooling is permitted. The end result of this is that more intracellular water will exit the RBCs, leading to less intracellular ice and less cellular damage.

The highest post-thaw RBC integrity observed with the slowfreezing conditions was with the two compounds that were the

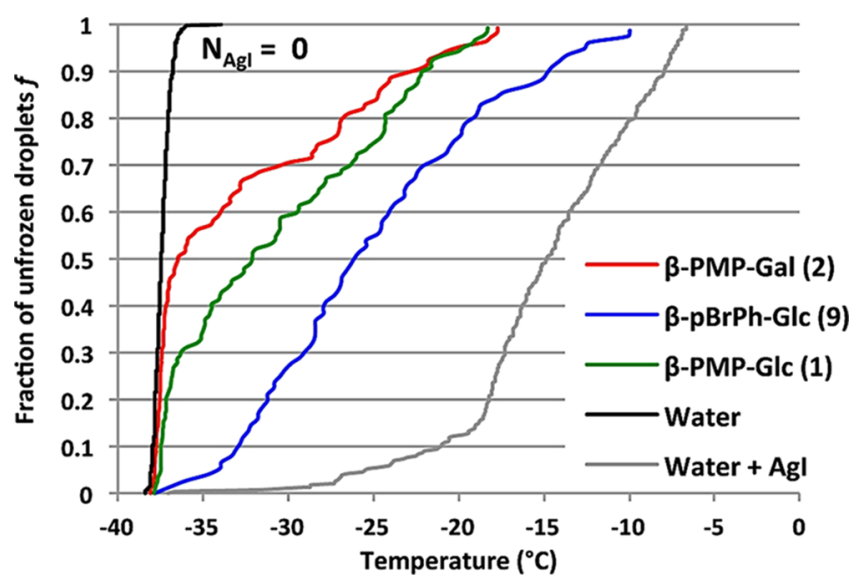

Figure 5. Fraction $f$ of unfrozen droplets for water and different aqueous solutions 1, 2, and 9 at $22 \mathrm{mM}$ with AgI particles $(5<d<25$ $\left.\mu \mathrm{m}, 2 \leq N_{\mathrm{AgI}} \leq 8\right)$ as a function of the sample temperature.

most potent ice recrystallization inhibitors, $\beta$-pBrPh-Glc (9) and $\beta$-pBrPh-Gal (22). The post-thaw integrity was $65 \%$ with $55 \mathrm{mM} \beta$-pBrPh-Gal (22) and $67 \%$ with $30 \mathrm{mM} \beta$-pBrPh-Glc (9). Both of these derivatives were also very effective with rapid freezing rates (Figure 4B). The addition of $30 \mathrm{mM}$ of either of these derivatives to $15 \%$ glycerol resulted in almost $95 \%$ postthaw integrity, which is significantly higher than that of $15 \%$ glycerol (75\% integrity). When the glycerol concentration was decreased to $10 \%$, $30 \mathrm{mM} \beta$-pBrPh-Glc (9) was still highly effective in preventing post-thaw hemolysis, resulting in almost triple the RBC integrity (67\%) compared to that of $10 \%$ glycerol (23\%).

\section{CONCLUSIONS}

Overall, the RBC freezing experiments demonstrate that IRIs $\beta$ PMP-Glc (1), $\beta$-pBrPh-Glc (9), and $\beta$-pBrPh-Gal (22) can be used at low concentrations in combination with $15 \%$ glycerol to provide high post-thaw $\mathrm{RBC}$ integrity with slow freezing rates that are used clinically to cryopreserve RBCs. Currently, this 
protocol requires $40 \%$ glycerol and time-consuming deglycerolization methods. Thus, significantly reducing glycerol concentrations can drastically improve post-thaw processing and allow for faster access to cryopreserved RBC units. The results presented in this article have highlighted how small structural changes to $\mathrm{O}$-aryl-glycosides modulate the ability of these compounds to inhibit ice recrystallization and alter cryoprotective ability. In addition, some of these compounds are potent inhibitors of ice nucleation. This study is the first detailed structure-function analysis that correlates changes in structure with the ability of a molecule to inhibit ice recrystallization and protect against cryoinjury during freezing. The $\mathrm{O}$-aryl-glycosides represent a novel class of small-molecule IRIs, many of which were effective cryoadditives used for the freezing of RBCs with reduced glycerol concentrations. The synthesis of these structurally simple molecules is amenable to large-scale preparation for use in cryopreservation applications to improve upon cryopreservation protocols of therapeutically important cells.

\section{METHODS}

All methods were carried out in accordance with approved guidelines.

Preparation of O-Aryl-Glycosides. Experimental procedures and spectroscopic data for the preparation of $O$-arylglycosides are described in the Supporting Information.

Ice Recrystallization Inhibition Activity. Ice recrystallization inhibition activity was measured using a "splat cooling" assay. ${ }^{39}$ All compounds were assessed in a PBS solution. The sample solution $(10 \mu \mathrm{L})$ was dropped from a micropipette onto a polished aluminum block precooled to $-80{ }^{\circ} \mathrm{C}$ through a tube two meters in height. The resulting frozen wafer was transferred to a cryostage held at $-6.4{ }^{\circ} \mathrm{C}$ to anneal for $30 \mathrm{~min}$. After the annealing time, a digital camera (Nikon CoolPix 5000) fitted to a microscope was used to photograph the ice crystals present in the wafer. For each wafer, three images were used to quantify the area of 12 ice crystals within the image ( $n$ $=36$ crystals per drop), and three drops of each sample were assayed ( $n=108$ per sample). A domain recognition software (DRS) program was used to analyze each image and employed a Microsoft Windows Graphical Interface to allow for the demarcation and storage of the vertices of ice domains in a digital micrograph to calculate the domain areas of each crystal. ${ }^{40}$ All data were plotted and analyzed using Microsoft Excel to provide the MGS of the sample and a PBS control on the same day of testing. The MGS of the sample was compared to that of the PBS control to obtain the ice recrystallization inhibition activity, reported as the percentage of the MGS (\% MGS) relative to the PBS control. A large \% MGS represents larger ice crystals, which is indicative of low IRI activity.

Blood Collection and Preparation. Canadian Blood Services Network Centre for Applied Development (NetCAD) provided all whole blood units, which were obtained in accordance with the standardized phlebotomy guidelines approved by Canadian Blood Services (CBS). Experimental protocols were approved by CBS and NetCAD, and ethics approvals were obtained from the Research Ethics Board (REB) at CBS and the University of Alberta. RBCs were obtained from whole blood units by centrifugation at $4{ }^{\circ} \mathrm{C}$ and $2200 \mathrm{~g}$ for $10 \mathrm{~min}$, followed by removal of the plasma supernatant and the buffy coat from the RBC pellet. Pelleted RBCs were washed twice with the $0.2 \% / 0.9 \%$ dextrose/saline buffer, then resuspended in an equal volume of packed RBCs in the $0.2 \% / 0.9 \%$ dextrose/saline buffer to a final hematocrit (Hct) of $0.50 \mathrm{~L} / \mathrm{L}$. Leukoreduced RBCs were stored refrigerated $\left(4-6{ }^{\circ} \mathrm{C}\right)$ in the buffer solution for up to 1 week.

RBC Freezing Experiments. Freezing solutions containing carbohydrate compounds were prepared at twice the desired final concentration in the $0.2 \% / 0.9 \%$ dextrose/saline buffer. The freezing solution $(150 \mu \mathrm{L})$ was added to $150 \mu \mathrm{L}$ of leukoreduced RBCs for a final volume of $300 \mu \mathrm{L}$. The final concentrations of all freezing solutions are indicated in the text. After transfer to cryotubes, the RBC suspensions were incubated for $10 \mathrm{~min}$ at room temperature, then immersed in a methanol bath cooled to $-5{ }^{\circ} \mathrm{C}$. A glycerol sample with a thermocouple was used as a control to measure the temperature of the solution in $1 \mathrm{~s}$ intervals. Once the internal solution reached $-5{ }^{\circ} \mathrm{C}$, ice nucleation was induced with forceps precooled in liquid nitrogen and the samples were held at $-5{ }^{\circ} \mathrm{C}$ for $5 \mathrm{~min}$. Slow freezing conditions were achieved by cooling at a rate of $1{ }^{\circ} \mathrm{C} / \mathrm{min}$ to $-40{ }^{\circ} \mathrm{C}$ in a methanol bath, followed by plunging the samples into dry ice $\left(-80{ }^{\circ} \mathrm{C}\right)$ and storing in a $-80{ }^{\circ} \mathrm{C}$ mechanical freezer. Rapid freezing conditions were achieved by plunging into dry ice $\left(-80{ }^{\circ} \mathrm{C}\right)$ and storing for a minimum of $30 \mathrm{~min}$.

Thawing and Post-Thaw Hemolysis. Fast-thaw conditions in a $37{ }^{\circ} \mathrm{C}$ water bath were used for all samples. Hcts and percentage hemolysis were assessed immediately post-thaw for all samples. The percentage post-thaw hemolysis (\% hemolysis) was determined using the cyanmethemoglobin Drabkin's method by comparing the supernatant hemoglobin concentration to total hemoglobin concentration. ${ }^{45,46}$ The percentage post-thaw RBC integrity was calculated using the following equation: \% post-thaw RBC integrity $=100 \%-\%$ hemolysis. Data for a minimum of four replicates for each condition are represented as the mean post-thaw RBC integrity.

Ice Nucleation Inhibition Assay. Compounds were assessed for ice nucleation inhibition using the ice-nucleating agent silver iodide (AgI) as previously described. ${ }^{47-49}$ Briefly, water-in-oil (W/O) emulsions containing AgI particles were prepared by suspending $0.2 \mathrm{~g}$ of $\mathrm{AgI}$ in $4 \mathrm{~mL}$ of purified deionized (DI) water in a glass vial. The suspension was sonicated for $60 \mathrm{~min}$ at room temperature and then immediately filtered through a filter paper to remove large AgI particles and aggregates, leaving only AgI particles of $\leq 3$ $\mu \mathrm{m}$ in diameter in the suspension. The aqueous solutions of each compound (except $\beta$-pBrPh-Glc 9) were prepared at three times the desired final concentration, and $1 \mathrm{~mL}$ of these aqueous solutions or water (control) was mixed with $2 \mathrm{~mL}$ of the AgI suspension. The aqueous solutions of $22 \mathrm{mM} \beta$-pBrPhGlc 9 were prepared at twice the desired final concentration because of the solubility limitation, and $1.5 \mathrm{~mL}$ of this aqueous solution was mixed with the same volume of the AgI suspension. The $3 \mathrm{~mL}$ mixtures were emulsified in $6 \mathrm{~mL}$ of $n$-heptane containing 4 wt $\%$ SPAN 65 in a glass vial. The ice nucleation temperature $T_{\mathrm{f}}$ was determined for each droplet of the $\mathrm{W} / \mathrm{O}$ emulsion by observing the freezing of the droplets by using optical microscopy. Before cooling, the droplet diameter $d$ was measured and the number of $\mathrm{AgI}$ particles $\left(\mathrm{N}_{\mathrm{AgI}}\right)$ inside of the droplet was counted. In this study, the data that satisfied $5<d<25 \mu \mathrm{m}$ and $2 \leq N_{\mathrm{AgI}} \leq 8$ were adopted.

\section{ASSOCIATED CONTENT}

\section{S Supporting Information}

The Supporting Information is available free of charge on the ACS Publications website at DOI: 10.1021/acsomega.6b00163. 
Experimental procedures and spectroscopic data for new compounds (PDF)

\section{AUTHOR INFORMATION}

\section{Corresponding Author}

*E-mail: rben@uottawa.ca. Phone: 1-613-562-5800.

\section{Notes}

The authors declare no competing financial interest.

\section{ACKNOWLEDGMENTS}

The authors gratefully acknowledge the Natural Sciences and Engineering Research Council of Canada (NSERC), Canadian Blood Services (CBS), and the Canadian Institutes of Health Research (CIHR) for financial support. C.J.C. thanks CBS for a Graduate Fellowship Program (GFP) award.

\section{REFERENCES}

(1) Hunt, C. J. Cryopreservation of human stem cells for clinical application: A review. Transfus. Med. Hemother. 2011, 38, 107-123.

(2) Harris, D. T. Non-haematological uses of cord blood stem cells. Br. J. Haematol. 2009, 147, 177-184.

(3) Scott, K. L.; Lecak, J.; Acker, J. P. Biopreservation of red blood cells: Past, present, and future. Transfus. Med. Rev. 2005, 19, 127-142.

(4) Hess, J. R. Red cell freezing and its impact on the supply chain. Transfus. Med. 2004, 14, 1-8.

(5) Hess, J. R.; Greenwalt, T. G. Storage of red blood cells: New approaches. Transfus. Med. Rev. 2002, 16, 283-295.

(6) Meryman, H. T. Cryopreservation of living cells: Principles and practice. Transfusion 2007, 47, 935-945.

(7) Meryman, H. T. Cryoprotective agents. Cryobiology 1971, 8, 173-183.

(8) McGann, L. E. Differing actions of penetrating and nonpenetrating cryoprotective agents. Cryobiology 1978, 15, 382-390.

(9) Fahy, G. M. The relevance of cryoprotectant "toxicity" to cryobiology. Cryobiology 1986, 23, 1-13.

(10) Davis, J. M.; Rowley, S. D.; Braine, H. G.; Piantadosi, S.; Santos, G. W. Clinical toxicity of cryopreserved bone marrow graft infusion. Blood 1990, 75, 781-786.

(11) Zambelli, A.; Poggi, G.; Da Prada, G. A.; Pedrazzoli, P.; Cuomo, A.; Miotti, D.; Perotti, C.; Preti, P.; Robustelli Della Cuna, G. Clinical toxicity of cryopreserved circulating progenitor cells infusion. Anticancer Res. 1998, 18, 4705-4708.

(12) Meryman, H. T.; Hornblower, M. A method for freezing and washing red blood cells using a high glycerol concentration. Transfusion 1972, 12, 145-156.

(13) Mazur, P. Cryobiology: The freezing of biological systems. Science 1970, 168, 939-949.

(14) Mazur, P. Freezing of living cells: Mechanisms and implications.

Am. J. Physiol. 1984, 247, C125-C142.

(15) Fowler, A.; Toner, M. Cryo-injury and biopreservation. Ann. N. Y. Acad. Sci. 2005, 1066, 119-135.

(16) Garner, J.; Harding, M. M. Design and synthesis of antifreeze glycoproteins and mimics. ChemBioChem 2010, 11, 2489-2498.

(17) Peltier, R.; Brimble, M. A.; Wojnar, J. M.; Williams, D. E.; Evans, C. W.; DeVries, A. L. Synthesis and antifreeze activity of fish antifreeze glycoproteins and their analogues. Chem. Sci. 2010, 1, 538-551.

(18) Gibson, M. I. Slowing the growth of ice with synthetic macromolecules: Beyond antifreeze(glyco) proteins. Polym. Chem. 2010, 1, 1141-1152.

(19) Balcerzak, A. K.; Capicciotti, C. J.; Briard, J. G.; Ben, R. N. Designing ice recrystallization inhibitors: From antifreeze (glyco)proteins to small molecules. RSC Adv. 2014, 4, 42682-42696.

(20) Capicciotti, C. J.; Doshi, M.; Ben, R. N. Ice recrystallization inhibitors: From biological antifreezes to small molecules. In Recent Developments in the Study of Recrystallization; Wilson, P., Ed.; InTech: New York, 2013; pp 177-224.
(21) Feeney, R. E.; Burcham, T. S.; Yeh, Y. Antifreeze glycoproteins from polar fish blood. Annu. Rev. Biophys. Biophys. Chem. 1986, 15, 59-78.

(22) Yeh, Y.; Feeney, R. E. Antifreeze proteins: Structures and mechanisms of function. Chem. Rev. 1996, 96, 601-618.

(23) Harding, M. M.; Anderberg, P. I.; Haymet, A. D. J. 'Antifreeze' glycoproteins from polar fish. Eur. J. Biochem. 2003, 270, 1381-1392.

(24) Wang, J.-H. A comprehensive evaluation of the effects and mechanisms of antifreeze proteins during low-temperature preservation. Cryobiology 2000, 41, 1-9.

(25) Carpenter, J. F.; Hansen, T. N. Antifreeze protein modulates cell survival during cryopreservation: Mediation through influence on ice crystal growth. Proc. Natl. Acad. Sci. U.S.A. 1992, 89, 8953-8957.

(26) Chao, H.; Davies, P. L.; Carpenter, J. F. Effects of antifreeze proteins on red blood cell survival during cryopreservation. J. Exp. Biol. 1996, 199, 2071-2076.

(27) Capicciotti, C. J.; Poisson, J. S.; Boddy, C. N.; Ben, R. N. Modulation of antifreeze activity and the effect upon post-thaw HepG2 cell viability after cryopreservation. Cryobiology 2015, 70, 7989.

(28) Liu, S.; Ben, R. N. C-linked galactosyl serine AFGP analogues as potent recrystallization inhibitors. Org. Lett. 2005, 7, 2385-2388.

(29) Czechura, P.; Tam, R. Y.; Dimitrijevic, E.; Murphy, A. V.; Ben, R. N. The importance of hydration for inhibiting ice recrystallization with C-linked antifreeze glycoproteins. J. Am. Chem. Soc. 2008, 130, 2928-2929.

(30) Leclère, M.; Kwok, B. K.; Wu, L. K.; Allan, D. S.; Ben, R. N. Clinked antifreeze glycoprotein (C-AFGP) analogues as novel cryoprotectants. Bioconjugate Chem. 2011, 22, 1804-1810.

(31) Gibson, M. I.; Barker, C. A.; Spain, S. G.; Albertin, L.; Cameron, N. R. Inhibition of ice crystal growth by synthetic glycopolymers: Implications for the rational design of antifreeze glycoprotein mimics. Biomacromolecules 2009, 10, 328-333.

(32) Inada, T.; Lu, S.-S. Thermal hysteresis caused by nonequilibrium antifreeze activity of poly(vinyl alcohol). Chem. Phys. Lett. 2004, 394, 361-365.

(33) Budke, C.; Koop, T. Ice recrystallization inhibition and molecular recognition of ice faces by poly(vinyl alcohol). ChemPhysChem 2006, 7, 2601-2606.

(34) Capicciotti, C. J.; Leclère, M.; Perras, F. A.; Bryce, D. L.; Paulin, H.; Harden, J.; Liu, Y.; Ben, R. N. Potent inhibition of ice recrystallization by low molecular weight carbohydrate-based surfactants and hydrogelators. Chem. Sci. 2012, 3, 1408-1416.

(35) Trant, J. F.; Biggs, R. A.; Capicciotti, C. J.; Ben, R. N. Developing highly active small molecule ice recrystallization inhibitors based upon C-linked antifreeze glycoprotein analogues. RSC Adv. 2013, 3, 26005-26009.

(36) Balcerzak, A. K.; Febbraro, M.; Ben, R. N. The importance of hydrophobic moieties in ice recrystallization inhibitors. RSC $A d v$. 2013, 3, 3232-3236.

(37) Capicciotti, C. J.; Kurach, J. D. R.; Turner, T. R.; Mancini, R. S.; Acker, J. P.; Ben, R. N. Small molecule ice recrystallization inhibitors enable freezing of human red blood cells with reduced glycerol concentrations. Sci. Rep. 2015, 5, 9692.

(38) Tam, R. Y.; Ferreira, S. S.; Czechura, P.; Chaytor, J. L.; Ben, R. N. Hydration index-a better parameter for explaining small molecule hydration in inhibition of ice recrystallization. J. Am. Chem. Soc. 2008, 130, 17494-17501.

(39) Knight, C. A.; Hallett, J.; DeVries, A. L. Solute effects on ice recrystallization: An assessment technique. Cryobiology 1988, 25, 5560.

(40) Jackman, J.; Noestheden, M.; Moffat, D.; Pezacki, J. P.; Findlay, S.; Ben, R. N. Assessing antifreeze activity of AFGP 8 using domain recognition software. Biochem. Biophys. Res. Commun. 2007, 354, 340344.

(41) Farrant, J.; Walter, C. A.; Lee, H.; McGann, L. E. Use of twostep cooling procedures to examine factors influencing cell survival following freezing and thawing. Cryobiology 1977, 14, 273-286. 
(42) Högman, C. F. Preparation and preservation of red cells. Vox Sang. 1998, 74, 177-187.

(43) Meryman, H. T. Frozen red cells. Transfus. Med. Rev. 1989, 3, 121-127.

(44) Rowe, A. W.; Eyster, E.; Kellner, A. Liquid nitrogen preservation of red blood cells for transfusion: A low glycerol-rapid freeze procedure. Cryobiology 1968, 5, 119-128.

(45) Drabkin, D. L. The standardization of hemoglobin measurement. Am. J. Med. Sci. 1948, 215, 110.

(46) Sowemimo-Coker, S. O. Red blood cell hemolysis during processing. Transfus. Med. Rev. 2002, 16, 46-60.

(47) Koyama, T.; Inada, T.; Kuwabara, C.; Arakawa, K.; Fujikawa, S. Anti-ice nucleating activity of polyphenol compounds against silver iodide. Cryobiology 2014, 69, 223-228.

(48) Inada, T.; Koyama, T.; Goto, F.; Seto, T. Ice nucleation in emulsified aqueous solutions of antifreeze protein type III and poly(vinyl alcohol). J. Phys. Chem. B 2011, 115, 7914-7922.

(49) Inada, T.; Koyama, T.; Goto, F.; Seto, T. Inactivation of ice nucleating activity of silver iodide by antifreeze proteins and synthetic polymers. J. Phys. Chem. B 2012, 116, 5364-5371. 